\title{
TUMOR NEUROENDÓCRINO DE ESTÓMAGO ASOCIADO A GLOMERULONEFRITIS RÁPIDAMENTE PROGRESIVA
}

\section{Viteri Cevallos, Diego y Martínez Ordaz, José Luis}

Servicio de Gastrocirugía. Centro Médico Nacional Siglo XXI - Instituto Mexicano del Seguro Social. Ciudad de México. México.

\begin{abstract}
Resumen:
Introducción: Los tumores neuroendocrinos gástricos son lesiones raras derivadas de células enterocromafines, cuya presentación depende de su patogenia y las características biológicas e histopatológicas, con una variedad amplia de presentación clínica, que amerita una visión integral para obtener un diagnóstico y manejo oportuno.

Caso Clínico: Se presenta el caso de una paciente femenina de 33 años que ingresa al servicio de Nefrología con diagnóstico de glomerulonefritis rápidamente progresiva, durante su evaluación clínica y estudios complementarios se encuentra un tumor neuroendócrino a nivel gástrico, se da manejo quirúrgico y se consigue mejoría clínica y a nivel de laboratorio con evolución favorable.

Conclusiones: La paciente presenta un síndrome paraneoplásico a nivel renal, secundario a un tumor neuroendócrino gástrico, sus reportes clínicos son raros en la literatura, ya que la paciente debutó con datos de una glomerulonefritis rápidamente progresiva, requirió un manejo multidisciplinario, que permitió corregir la causa del padecimiento, aunque con el riesgo de daño renal crónico a largo plazo.
\end{abstract}

Palabras clave: tumor neuroendócrino gástrico, glomerulonefritis.

Recibido: 8 Enero 2015. Aceptado: 24 Marzo 2015. Publicado: 15 Abril 2015.

Revista electrónica publicada por el Departamento de Farmacología de la Escuela de Medicina de la Universidad de Costa Rica, 2060 San José, Costa Rica. ${ }^{\circledR}$ All rights reserved. Licensed under a Creative Commons Unported License. 


\title{
GASTRIC NEUROENDOCRINE TUMOR ASSOCIATED WITH RAPIDLY PROGRESSIVE GLOMERULONEPHRITIS
}

\begin{abstract}
Introduction: Gastric neuroendocrine tumors are rare lesions derived from enterochromaffin cells, whose presentation depends on its pathogenesis and biological and histopathological characteristics, with a wide variety of clinical presentation, which deserves an integral vision for a diagnosis and timely managment. Case Report: This is the case of a 33 years old woman who enters to the Nephrology Service diagnosed with rapidly progressive glomerulonephritis, during her clinical evaluation and complementary studies, a neuroendocrine tumor was found at the gastric level, the patient received surgical management and a clinical improvement is achieved as well as the laboratory with favorable outcome.

Conclusions: A paraneoplasic syndrome is presented, secondary to a neuroendocrine gastric tumor, the medical reports are very rare in the literature, since the patient presented data from a rapidly progressive glomerulonephritis. She required a multidisciplinary management in which was possible to correct the cause of her suffering, but long term with the risk of chronic renal damage.
\end{abstract}

Key words: gastric neuroendocrine tumor, glomerulonephritis (MESH).

\section{INTRODUCCIÓN}

Los tumores neuroendocrinos gástricos (GNETs) son lesiones raras que se caracterizan por hipergastrinemia, son derivados de células enterocromafines, consisten en un grupo heterogéneo de neoplasias que comprenden diferentes tipos de tumores, con diferente patogenia, características histomorfológicas $\mathrm{y}$ comportamiento biológico [1].

Estas neoplasias, en los últimos 50 años, han presentado aumento en la mayoría de los países, en parte debido a una mayor conciencia y un uso más generalizado de la endoscopia gastrointestinal superior [2].

Los síndromes paraneoplásicos son una serie de complejos síntomas que se manifiestan sistémicamente como el resultado de la producción de hormonas, factores de crecimiento, citoquinas y otras. Un número significativo de estos síndromes son causados por los productos de secreción, hormonas peptídicas, principalmente de células neuroendócrinas, que están ampliamente dispersos en el pulmón, gastrointestinal, páncreas, glándula tiroides, médula adrenal, piel, próstata y mama [3].

\section{CASO CLÍNICO}

Paciente femenino de 33 años de edad originaria del Distrito Federal, estudios de nivel superior, soltera, carga genética materna para artritis reumatoide y diabetes mellitus, abuelos maternos con cáncer de páncreas y esófago. AGO: menarca a los 15 años, ciclos regulares, FUM 02.07.14, colposcopia hace 6 meses con diagnóstico de infección por VPH con tratamiento local no especificado. APP: Síndrome de Sjögren de 6 años de diagnóstico con tratamiento sintomático, hipotiroidismo en manejo con levotiroxina $50 \mathrm{mg}$ VO cada $24 \mathrm{hrs}$, trombosis venosa profunda en manejo con anticoagulación oral. Alergia a ciprofloxacino y sulfas. 
Antecedentes quirúrgicos: Rinoplastia secundaria a traumatismo hace 10 años.

\section{PADECIMIENTO ACTUAL}

Inicia hace aproximadamente 5 meses posterior a viaje de larga distancia con aumento de volumen en miembros pélvicos de predominio en zonas de presión y declive, asociado a petequias ocasionales, refiere además dolor tipo ardoroso en plantas de los pies, malestar general, astenia, adinamia y alopecia, por lo que recibe tratamiento homeopático no especificado. No presenta mejoría, acude a valoración a nuestro hospital, se documenta falla renal aguda y se decide ingreso a cargo del servicio de nefrología para la realización de hemodiálisis y protocolo diagnóstico con sospecha de glomerulonefritis rápidamente progresiva.

En el interrogatorio dirigido refiere distensión abdominal, intolerancia a la vía oral ocasional, náuseas sin llegar a emesis, niega diarrea. Niega pérdida de peso. Síntomas mejoran con la restricción de alimentos. Además refiere xeroftalmia, aumento de volumen ocular bilateral, mialgias y artralgias.

\section{EXAMEN FÍSICO}

Signos vitales TA $110 / 70$, FC 80, T $^{\circ} 7^{\circ} \mathrm{C}$. Paciente consciente, orientada, cardiorrespiratorio sin datos de compromiso, adecuada entrada y salida de aire. Ruidos cardiacos rítmicos de buen tono y de buena intensidad. Abdomen se encuentra plano, blando, depresible, peristalsis presente, sin datos de megalias, niega dolor a la palpación. Sin datos de irritación peritoneal. Extremidades íntegras con adecuada conformación, sin evidencia de datos de compromiso vascular. Pulsos pedios presentes. Resto de la exploración sin datos patológicos.

\section{ESTUDIOS COMPLEMENTARIOS}

Resultados de estudios de ingreso

\begin{tabular}{|c|c|c|}
\hline Urea & Creatinina & Depuración creatinina \\
\hline $\begin{array}{c}83 \mathrm{mg} / \mathrm{dl} \\
(20-40)\end{array}$ & $\begin{array}{c}3.4 \mathrm{mg} / \mathrm{dl} \\
(0.8-1)\end{array}$ & $\begin{array}{c}19.6 \mathrm{ml} / \mathrm{minuto} \\
(97-137)\end{array}$ \\
\hline & & \\
Proteínas & Creatinina & Resto de estudios \\
orina $24 \mathrm{~h}$ & urinaria & iniciales \\
\hline $3.4 \mathrm{~g} / 24 \mathrm{~h}$ & $\mathbf{4 6} \mathrm{g} / 24 \mathrm{~h}$ & normales \\
$(<30 \mathrm{mg} / 24 \mathrm{~h})$ & $(1-1.6)$ & \\
\hline
\end{tabular}

Posteriormente durante protocolo de estudio de su nefropatía se realizan los siguientes estudios reumatológicos especiales.

Resultados de estudios reumatólogicos

\begin{tabular}{|c|c|c|}
\hline $\begin{array}{c}\text { Anticuerpo } \\
\text { Anticardiolipina }\end{array}$ & $\begin{array}{c}\text { Ac Anti SSA } \\
(\text { Ro) }\end{array}$ & Ac Antinuclear \\
\hline $2.2 \mathrm{U} / \mathrm{ml}(-)$ & $10 \mathrm{U} / \mathrm{ml}(-)$ & $0.6 \mathrm{U} / \mathrm{ml}(-)$ \\
\hline $\begin{array}{c}\text { Ac Anti DNA } \\
\text { doble cadena }\end{array}$ & $\begin{array}{c}\text { Anticoag. } \\
\text { Lúpico }\end{array}$ & $\begin{array}{c}\text { Ac } \\
\text { Antifosfolípidos }\end{array}$ \\
\hline $17 \mathrm{U} / \mathrm{ml}(-)$ & $(-)$ & $(-)$ \\
\hline
\end{tabular}

Resultados estudios hormonales

\begin{tabular}{|c|c|c|}
\hline Prolactina & Gastrina & TSH \\
\hline $351.7 \mathrm{ng} / \mathrm{ml}$ & $3702 \mathrm{pg} / \mathrm{ml}$ & $1.86 \mathrm{mUl} / \mathrm{L}$ \\
$(3.4-24.1)$ & $(13-115)$ & $(0.27-4.2)$ \\
\hline & & \\
$\mathrm{T} 4$ & $\mathrm{PTH}$ & Acido fólico \\
\hline $1.17 \mathrm{ng} / \mathrm{dl}$ & $141 \mathrm{pg} / \mathrm{ml}$ & $11.23 \mathrm{ng} / \mathrm{ml}$ \\
$(0.930-1.7)$ & $(10-(65)$ & $(3.1-17.5)$ \\
\hline
\end{tabular}

Resultados de estudios inmunológicos

\begin{tabular}{|l|l|l|}
\hline FR & C3 & C4 \\
\hline $11.9 \mathrm{UI} / \mathrm{ml}$ & $88.8 \mathrm{mg} / \mathrm{dl}$ & $28.9 \mathrm{mg} / \mathrm{dl}$ \\
$(0.0-14)$ & $(90-180)$ & $(10-40)$ \\
\hline $\mathrm{IgM}$ & lgG & lgA \\
\hline $197 \mathrm{mg} / \mathrm{dl}$ & $1073 \mathrm{mg} / \mathrm{dl}$ & $240 \mathrm{mg} / \mathrm{dl}$ \\
$(40-230)$ & $(100-1600)$ & $(70-400)$ \\
\hline
\end{tabular}


Con los estudios previos se realiza valoración por el servicio de Reumatología sin encontrar datos clínicos y/o bioquímicos de síndrome de Sjögren, Schimer ( - ), Anti Ro y Anti La (negativos). No cumple criterios para síndrome antifosfolípidos, sin datos de vasculitis. Valoración por Endocrinología sin evidencia de patología hipofisaria (por clínica y por imagen). Cuenta con hiperprolactinemia secundario a enfermedad renal y uso de metoclopramida.

Por los datos bioquímicos de hipergastrinemia se decide realizar una panendoscopia con los siguientes hallazgos:
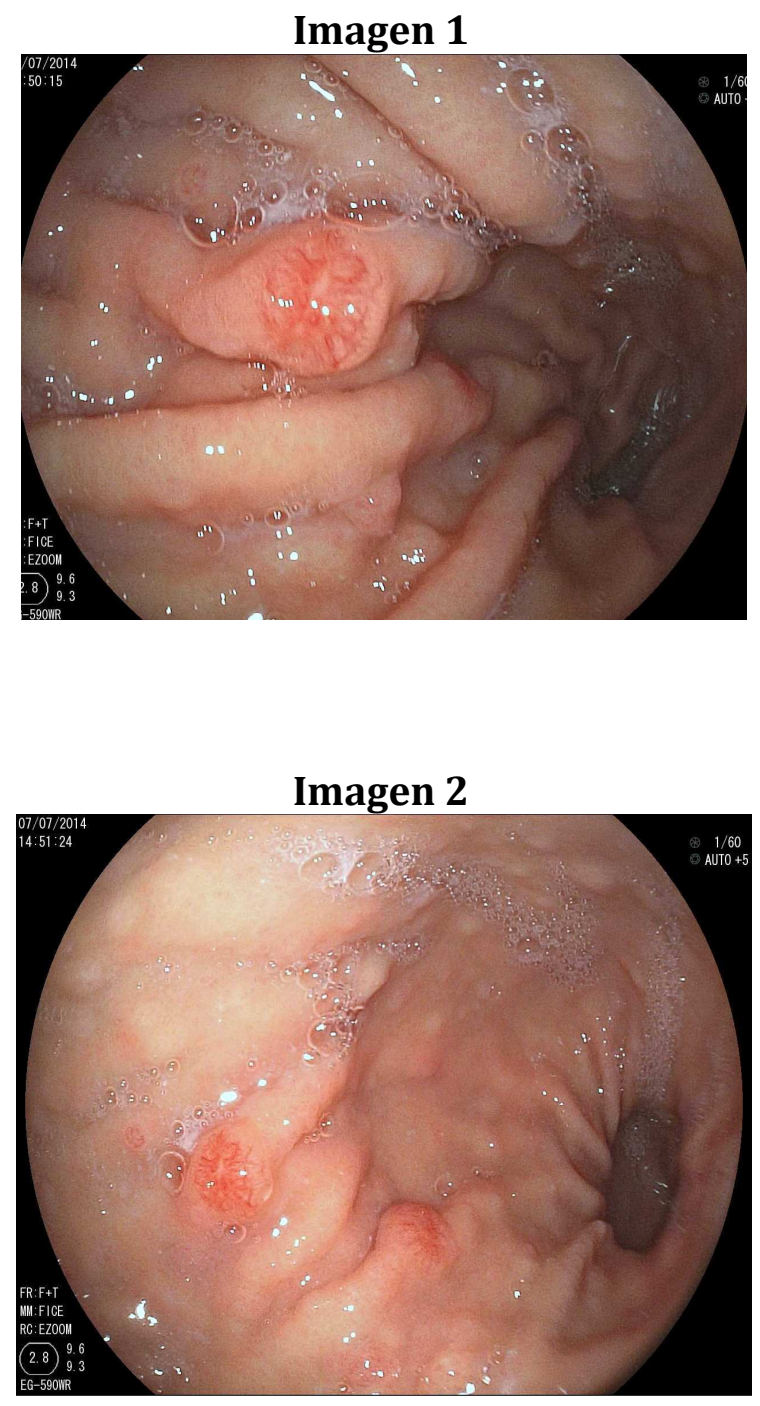

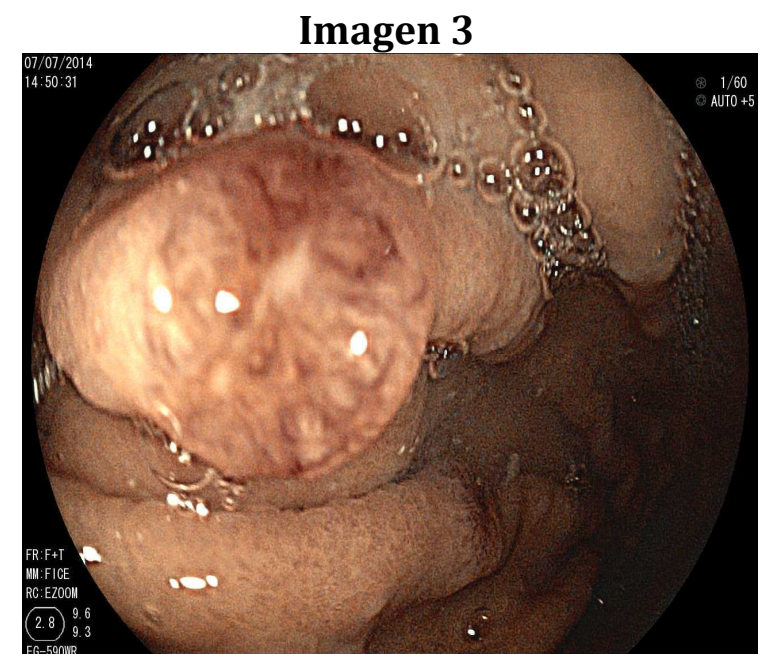

Imagen 1, 2 y 3. Visión macroscópica de pólipos en curvatura mayor de estómago por endoscopia.

En estómago se evidencia la mucosa del cuerpo con patrón folicular, en curvatura mayor se observan 3 pólipos de $7-10 \mathrm{~mm}$, uno de ellos con centro de fibrina, con patrón glandular tubular largo. No resecables. Diagnósticos: Gastropatía folicular de cuerpo, pólipos de cuerpo medio. Se toman biopsias.

Biopsia Estudio No: B-14-2962: Pólipos Gástricos. Informe IHQ cromogranina positiva $3+/ 3+$, sinaptofisina positiva $3+/ 3+$, Ki 67,2 , CD 117 negativo, PS100 negativo, CD 34 negativo. Dx de Neoplasia Neuroendócrina de Bajo Grado.

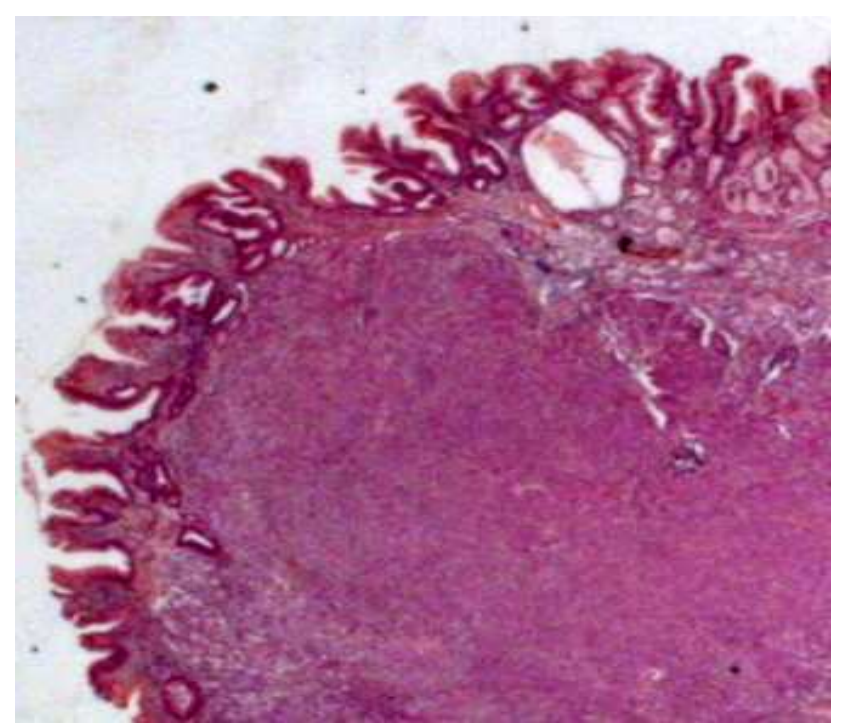

Imagen 4. Imagen microscópica de biopsia de pólipo.

Revista electrónica publicada por el Departamento de Farmacología de la Escuela de Medicina de la Universidad de Costa Rica, 2060 San José, Costa Rica. ${ }^{\circledR}$ All rights reserved. Licensed under a Creative Commons Unported License. 
Se realizó una tomografía de abdomen sin evidencia tumores o de adenopatías.

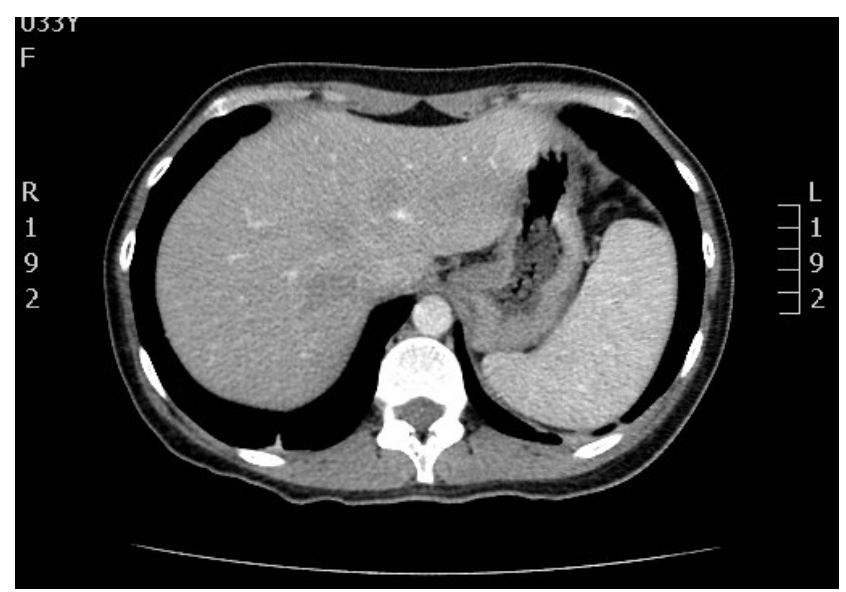

Imagen 5. TC contrastada de abdomen.

\section{MANEJO Y EVOLUCIÓN}

Se realiza interconsulta al servicio de Gastrocirugía, se decide tratamiento quirúrgico, se realiza una gastrectomía en cuña con resección macroscópica de los 3 pólipos a nivel de cara anterior de cuerpo gástrico. Evolución quirúrgica favorable sin complicaciones con normalización de niveles de gastrina y seguimiento por parte de Nefrología por riesgo de insuficiencia renal crónica a largo plazo.

\section{DISCUSIÓN}

Los tumores neuroendócrinos (TNE) son tumores raros que surgen de células progenitoras, pueden localizarse en los pulmones (20\%) y muy raramente en la mama, ovario y próstata, la mayoría están a nivel gastrointestinal, los cuales, generalmente no producen péptidos u hormonas. El 25\% están en intestino delgado, colon y recto (15\%) y páncreas (7\%). Los TNE gástricos representan el $5 \%$ de todos los TNE y se diagnostican a menudo por casualidad durante la endoscopia superior con síntomas no relacionados.
TNE gástricos se pueden dividir en 3 tipos:

- I (80\%) se asocian con aclorhidria e hipergastrinemia, en pacientes con gastritis atrófica crónica, se presentan como lesiones pequeñas $(2-10 \mathrm{~mm})$, sésiles y múltiples, las lesiones macroscópicas están ausentes y se diagnostica por biopsia, estos tumores suelen ser bien diferenciados y con Ki67 baja, las METS son raras.

- Tipo II (5\%) están asociados con neoplasia endocrina múltiple (NEM) tipo I y en menor medida con el síndrome de Zollinger-Ellison, presentan crecimiento infiltrante y diseminación metastásica

(10-30\%).

- Tipo III son tumores esporádicos y representan el $15-25 \%$ de todos los TNE gástricos, son generalmente solitarios y mayores a $2 \mathrm{~cm}$. Causan anemia por déficit de hierro y dolor abdominal, son invasivos con METS del 50 al 100\%.

En pacientes con TNE se pueden presentar síndromes paraneoplásicos a nivel cutáneo, endócrino, hematológico, neurológico, reumatológico y ocular. Las manifestaciones renales son raras, solo existen varios reportes de casos en que estos tumores se asocian a síndrome nefrótico, la lesión más común es la glomerulonefritis membranosa.

\section{CONCLUSIÓN}

Se presenta el reporte de un caso, cuyos reportes clínicos son raros en la literatura, con diagnóstico de un tumor neuroendócrino a nivel de estómago, que debutó con datos de una glomerulonefritis rápidamente progresiva, requirió un manejo multidisciplinario, que permitió corregir la causa del padecimiento, aunque con el riesgo de daño renal crónico a largo plazo.

\section{BIBLIOGRAFÍA}

1. Li T, Qiu F, Qian Z, Wan J, Qi X, Wu B. Classification, clinicopathologic features and treatment of gastric neuroendocrine tumors. World J Gastroenterol 2014; 20:118-125. 
2. Chen WF, Zhou PH, Li QL, Xu MD, Yao LQ. Clinical impact of endoscopic submucosal dissection for gastric neuroendocrine tumors: a retrospective study from mainland China. Scientific World J. 2012;2012:869769.

3. Kaltsas G, Androulakis I, Herder, Grossman A. Paraneoplasic syndromes secondary to neuroendocrine tumours. Endocrine-Related Cancer 2010,17:173-193.

4. Ramage J, Ahmed A, Ardill J. UK and Ireland Neuroendocrine Tumour Society. Guidelines for the Management of Gastroenteropancreatic Neuroendocrine (Including Carcinoid) Tumours (NETs). Gut 2012;61:6-32.

5. Hemminki K, Li X. Incidence Trends and Risk Factors of Carcinoid Tumors: A Nationwide Epidemiologic Study from Sweden. Cancer 2001;92:2204-2210.

6. Vannella L, Lahner E, Annibale B. Risk for Gastric Neoplasias in Patients with Chronic Atrophic Gastritis: A Critical Reappraisal. World J Gastroenterol 2012;18:1279-1285.

7. Vannella L, Sbrozzi-Vanni A, Lahner E.Development of Type I Gastric Carcinoid in Patients with Chronic Atrophic Gastritis. Aliment Pharmacol Ther 2011;33:1361-1369.

8. Van Weywnberg S, Pohl J. Gastric Neuroendocrine Tumors. Video Journal and Encyclopedia of GI Endoscopy 2013;1:176-177.

\section{INFORMACIÓN DEL AUTOR}

Viteri Cevallos, Diego Fernando

diegoviteri83@hotmail.com 